\title{
Magnetic clouds, cosmic ray decreases, and geomagnetic storms
}

\author{
S. O. Ifedili \\ Department of Physics, University of Benin, Benin City, Nigeria \\ (Received March 24, 2005; Revised October 8, 2005; Accepted October 8, 2005; Online published May 12, 2006)
}

\begin{abstract}
The relationship between magnetic clouds, cosmic ray decreases and geomagnetic storms has been investigated by using some cosmic ray hourly intensities recorded with ground-based monitors at Alert, Deep River and Mount Washington, as well as the geomagnetic activity Dst index, and the interplanetary magnetic field (IMF) and the solar wind plasma (SWP) bulk-speed, density and temperature in the near-Earth space, on 28-30 September 1978, 24-26 April 1979, 13-15 January 1967, 3-5 January 1978, and 27-29 November 1989. Due to the interplanetary coronal mass ejection (ICME) impacting on slow solar wind, there is a sheath upstream of the ICME led by a fast forward shock. And the large IMF variations in this sheath, which sustain the depressions in the cosmic ray intensity during Forbush decreases (FDs), were found not to influence the main phase storm, but rather the southward IMF in the said sheath and magnetic cloud was the major source in triggering geomagnetic storms, by allowing a strong coupling between the solar wind and the magnetosphere. It was also observed that the initial set of the main phase storm always began in the sheath where, and when, the sustained southward-oriented IMF first occurred, but ceased when the IMF was rotated to a strong northward-orientation, only to resume at subsequent sustained southward-oriented IMF within the sheath and the leading (i.e., front) region of the magnetic cloud. The front boundary of the magnetic cloud was found to be well defined by the relatively high ( $\gtrsim 10 \mathrm{nT}) \mathrm{rms}$ of the IMF components, which prominently separates both the Lull region of the sheath and the onset of the second decrease of the two-step FD, from the magnetic cloud. There were some instances where a two-step main phase storm, caused by the combination of a sheath and cloud structure, occurred, the two steps sometimes both starting in the sheath itself. Also, in some cases, the sheath and the leading region of the magnetic cloud together produced a single-step storm. In addition, enhanced IMF south latitude and IMF intensity in the sheath and magnetic cloud during the IMF sustained southern orientation, were each observed to produce enhanced geomagnetic activity, even for intense storms. And high SWP bulk speed was found to reduce the depth of the Dst index. Therefore, it appears that when the magnetosphere is exposed to a sustained southward-oriented IMF in the magnetic cloud and the sheath preceding it, a valve (i.e., valve-like IMF direction) opens and allows direct transfer of energy between the solar wind and the magnetosphere to trigger the geomagnetic storms, such that the stronger the sustained IMF south-ward orientation, the wider the valve opens, the higher the SWP bulk speed, the narrower the opening in the valve becomes. And the more the IMF strength during the IMF southern orientation, the larger is the solar wind energy density that is available for transfer through the valve. The valve closes when the IMF is rotated to a strong northward-orientation, and the geomagnetic storms cease.

Index terms: 2104 Interplanetary Physics: Cosmic rays; 2111 Interplanetary Physics: Ejecta, driver gases, and magnetic clouds; 2139 Interplanetary Physics: Interplanetary shocks; 7513 Solar Physics, Astrophysics, and Astronomy: Coronal mass ejections.
\end{abstract}

Key words: Cosmic ray modulation, magnetic clouds, interplanetary shocks, coronal mass ejections, geomagnetic storms, space weather.

\section{Introduction}

Coronal mass ejections (CMEs) produce large disturbances in the solar wind and they generally reach the nearEarth space inside the so-called high-speed plasma streams (see, for instance, Storini, 1990). These disturbances are the primary cause of geomagnetic storms (e.g., Gosling, 1993a, b). On the other hand, the two-step Forbush decrease (FD) in cosmic ray records seems to originate from the structure within the shock and sheath preceding the interplanetary coronal mass ejection (Ifedili, 2001, 2004). Moreover, magnetic clouds pertain to a subset of CMEs

Copyright (c) The Society of Geomagnetism and Earth, Planetary and Space Sciences (SGEPSS); The Seismological Society of Japan; The Volcanological Society of Japan; The Geodetic Society of Japan; The Japanese Society for Planetary Sciences; TERRAPUB. in the interplanetary space (ICMEs) with characteristic features well described in the past (e.g., Burlaga et al., 1981; Klein and Burlaga, 1982; Zhang and Burlaga, 1988; Iucci et al., 1989; Bavassano et al., 1989).

In this paper, we investigate the relationship between magnetic clouds, cosmic ray decreases, and geomagnetic storms for the following time intervals when the FD amplitude was $>4 \%$ at Alert station where the diurnal variation is not enhanced: September 28-30, 1978, April 24-26, 1979, January 13-15, 1967, January 3-5, 1978, and November 27-29, 1989.

For each solar wind stream overcoming the terrestrial environment, we looked for the shock, sheath and magnetic cloud positions. This was done as follows: 
- Shock: The arrival of the interplanetary shock waves at the Earth, i.e., fast forward shocks, was identified by the sudden increase of the IMF intensity and the SWP bulk speed, density, and temperature in the near-Earth space solar wind data, and as the time of the storm sudden commencement (SSC).

- Sheath: A sheath exists upstream of the ICME led by a fast forward shock; the sheath material is slow solar wind that has been swept up by the ICME and is not part of the ICME itself. The sheath is sufficiently turbulent as indicated by the large variations in both the field strength and direction as well as by the relatively high ( $\gtrsim 10 \mathrm{nT}) \mathrm{rms}$ of the IMF components in the region; the sheath has a relatively high speed, is hot $\left(\gtrsim 10^{5} \mathrm{~K}\right)$ and dense $\left(\gtrsim 10 \mathrm{~cm}^{-3}\right)$ and was most probably produced by the shock propagation in space (Ifedili, 2004, and references therein).

- Lull Region: This is the middle region of the sheath that coincides with the recovery phase of the first step of the two-step FD, and in which there are relatively low IMF variations as well as a relatively low $(\lesssim 5 \mathrm{nT})$ rms of the IMF components (Ifedili, 2001, 2004).

- Magnetic Cloud: This is the region of IMF rotation (IMF direction changes smoothly from large southern (northern) direction to large northern (southern) direction) and elevated IMF intensity ( $\gtrsim 10 \mathrm{nT})$, and relatively low SWP density and temperature $\left(\lesssim 10^{4} \mathrm{~K}\right)$ and relatively low $(\lesssim 1 \mathrm{nT}) \mathrm{rms}$ of the IMF components (e.g., Burlaga et al., 1981; Klein and Burlaga, 1982; Zhang and Burlaga, 1988; Iucci et al., 1989; Bavassano et al., 1989; Ifedili, 2004). Our estimated start and end times of the magnetic cloud in Fig. 1 as well as the start time of the magnetic cloud in Fig. 4 are the same as by Zhang and Burlaga (1988), while our estimated start and end times of the magnetic cloud in Fig. 2 are the same as by Cane et al. (1994).

\section{Data Used}

In our work we used the following data:

(i) Hourly values of the solar wind plasma (SWP) bulk speed $(\mathrm{V})$, density $(\mathrm{N})$, temperature $(\mathrm{T})$, and interplanetary magnetic field (IMF) magnitude (F) and latitude $(\Theta)$, taken from King (1977, 1983), and King and Papitashvili (1994) (See also the National Space Data Centre (NSSDC) CDAWeb at http://cdaweb.gsfc.nasa.gov); and of the rms of the IMF components (c) taken from NSSDC CDAWeb (http://cdaweb.gsfc.nasa.gov), and NSSDC OMNIWeb (http://nsdc.gsfc.nasa.gov/omniweb/ow.html)

(ii) Hourly cosmic ray intensities registered by three ground-based neutron monitors (and provided by Prof. J. A. Lockwood, Drs. G. Dolling (Atomic Energy of Canada Limited) and E. Erwin (National Geophysical Data Centre, U.S.A.)) for FD events with amplitude $>4 \%$ at Alert station where the diurnal variation is not enhanced:

Alert (geographic coordinates: $\mathrm{N} 82^{\circ} 30^{\prime} \mathrm{E} 297^{\circ} 40^{\prime}$, rigidity cutoff $P_{c}=0.00 \mathrm{GV}$, height $h=0.00 \mathrm{~m}$ (sea level), years: $1967,1978,1979$; station was closed in 1987.)
Deep River (geog. coord.: N46 $36^{\prime}$ E282 $30^{\prime}, P_{c}=1.02$ $\mathrm{GV}, h=145$ m, years: 1967, 1978, 1979, 1989)

Mount Washington (geog. coord.: N44 $18^{\prime}$ E288 $42^{\prime}$, $P_{c}=1.24 \mathrm{GV}, h=1900 \mathrm{~m}$, years: $1967,1978,1979$, 1989)

(iii) The hourly geomagnetic Dst index and the SSC occurrence, respectively taken from CDAWeb at http://cdaweb.gsfc.nasa.gov, and ftp:// ftp:ngdc.noaa.gov/STP/SOLAR_DATA/SUDDEN_ COMMENCEMENTS/STORM2.S.

Figures $1-5$ show the hourly averages of the cosmic ray intensity at Alert, Deep River, and Mount Washington as well as of the IMF (magnitude and latitude) and the SWP bulk speed, density, and temperature, the rms of the IMF components $\left(\sigma_{c}\right)$ and the geomagnetic activity Dst index, at $1 \mathrm{AU}$, as listed in CDAWeb/OMNIWeb, during the periods, 28-30 September 1978 (Fig. 1), 24-26 April 1979 (Fig. 2), 13-15 January 1967 (Fig. 3), 3-5 January 1978 (Fig. 4), and 27-29 November 1989 (Fig. 5). In the figures, the arrival of interplanetary shock waves at the Earth was identified by

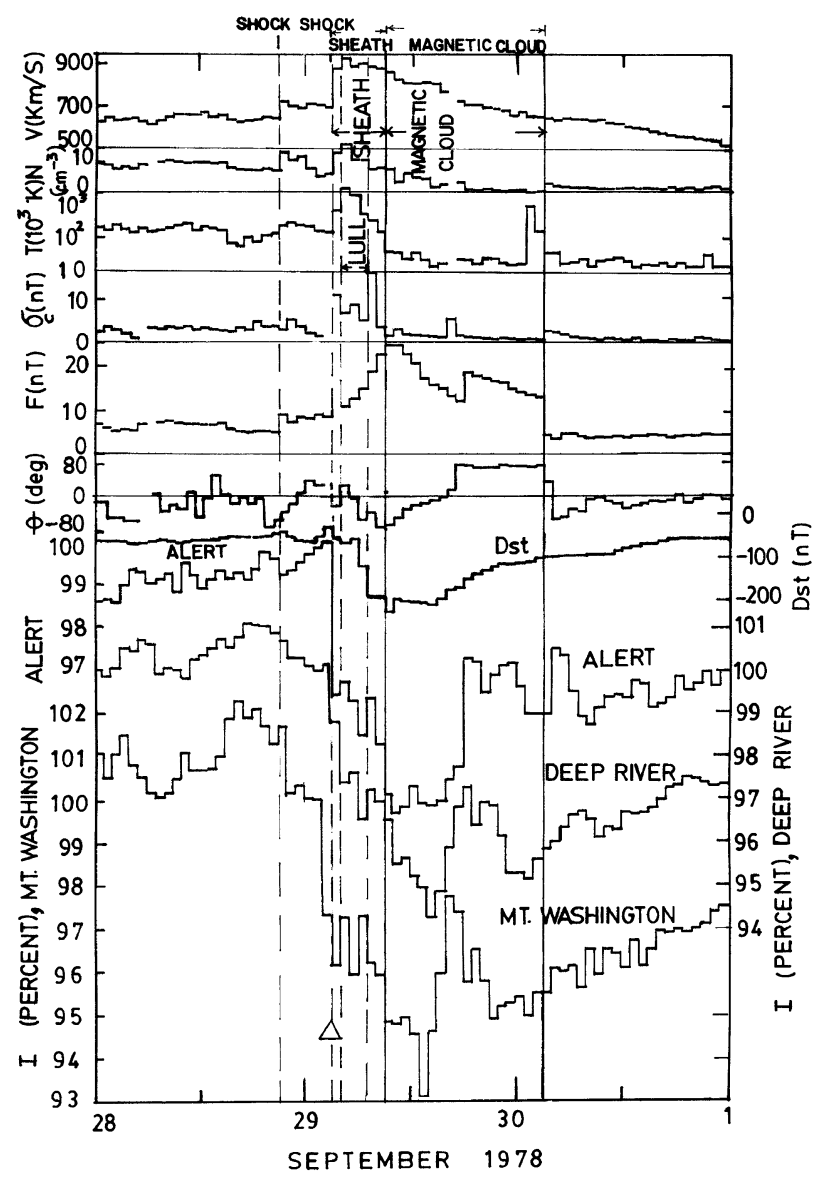

Fig. 1. Hourly profiles of the Alert, Deep River and Mount Washington nucleonic intensity (I), bulk-speed (V), density (N) and temperature (T) of the solar wind plasma, interplanetary magnetic field (IMF) magnitude (F) and latitude $(\theta)$ as well as the rms of the IMF components $\left(\sigma_{c}\right)$ and the geomagnetic activity Dst index on 28-30 September 1978. Storm sudden commencement is marked by a triangle, and the vertical dashed line, labeled SHOCK, marks the arrival of the interplanetary shock wave at the Earth. The single-step Dst main phase storm is due to the combination of the sheath and the leading field of the magnetic cloud. 


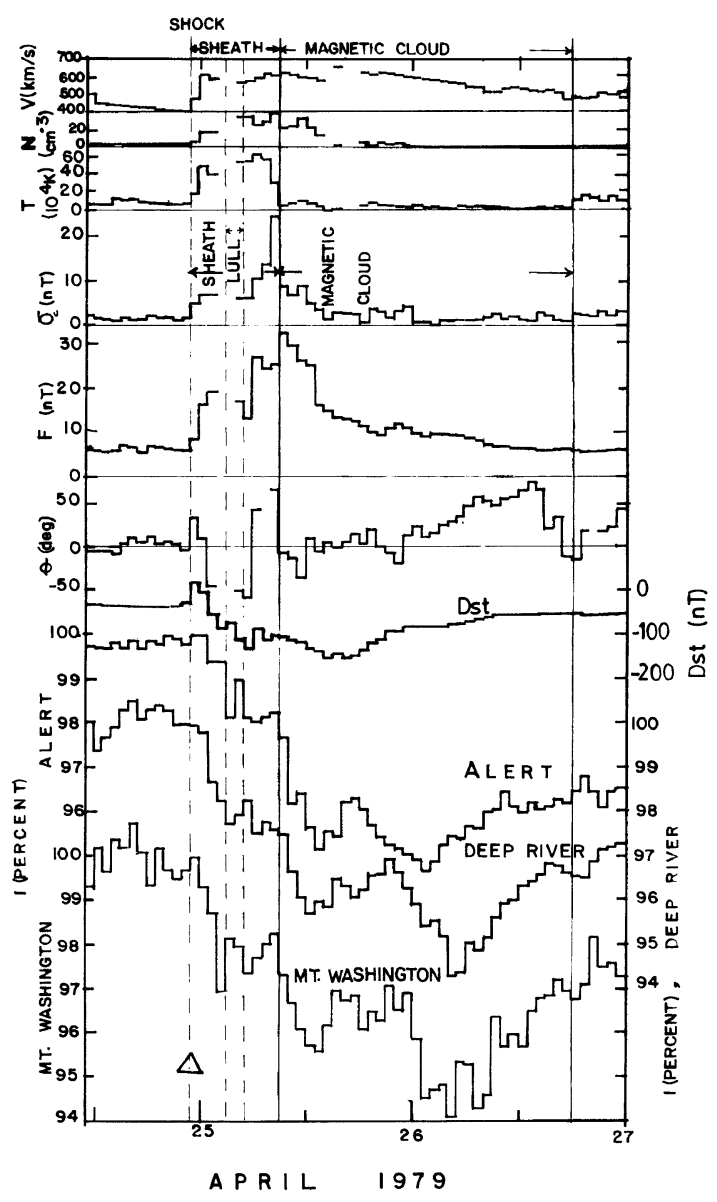

Fig. 2. Same as Fig. 1, but for 24-26 April 1979 and the first step of the two-step main phase storm which is due to the sheath, and the second step which is due to the leading field of the magnetic cloud.

the sudden increase of the IMF intensity and the SWP bulk speed, density, and temperature, as well as the time of the SSC. They occurred at $\sim 2200$ UT on 28 September 1978, at $\sim 0400$ UT on 29 September 1978, at $\sim 2358$ UT on 24 April 1979, at $\sim 1300$ UT on 13 January 1967, at $\sim 2042$ UT on 3 January 1978, at 2139 UT on 27 November 1989, and at $\sim 0900$ UT on 28 November 1989, which approximately coincided with the onset time of the FDs at Alert, Deep River and Mount Washington, and which are represented by the vertical dashed line labeled SHOCK, in Figs. 1-5, that indicates the approximate time of arrival of the fast forward shock at $1 \mathrm{AU}$.

\section{Data Analysis and Results}

Table 1 summarizes the obtained results for the investigated events. The first eight columns in Table 1 respectively give the figure number, the date, the relevant shock time, the estimated start and end times of the magnetic cloud, the maximum SWP bulk speed as well as the Forbush decrease amplitude for the total decrease and the second decrease at the Alert station, where the diurnal variation is not enhanced. Notice that Mount Washington station is used in Table 1 for Fig. 5, the November 1989 event, since the Alert station was closed in December 1987, and that the FD amplitude $(\%)$ in Table 1 is calculated as $100\left(N_{O}-N_{F}\right) / N_{O}$, where $N_{O}$ is the hourly average counting rate of the de-

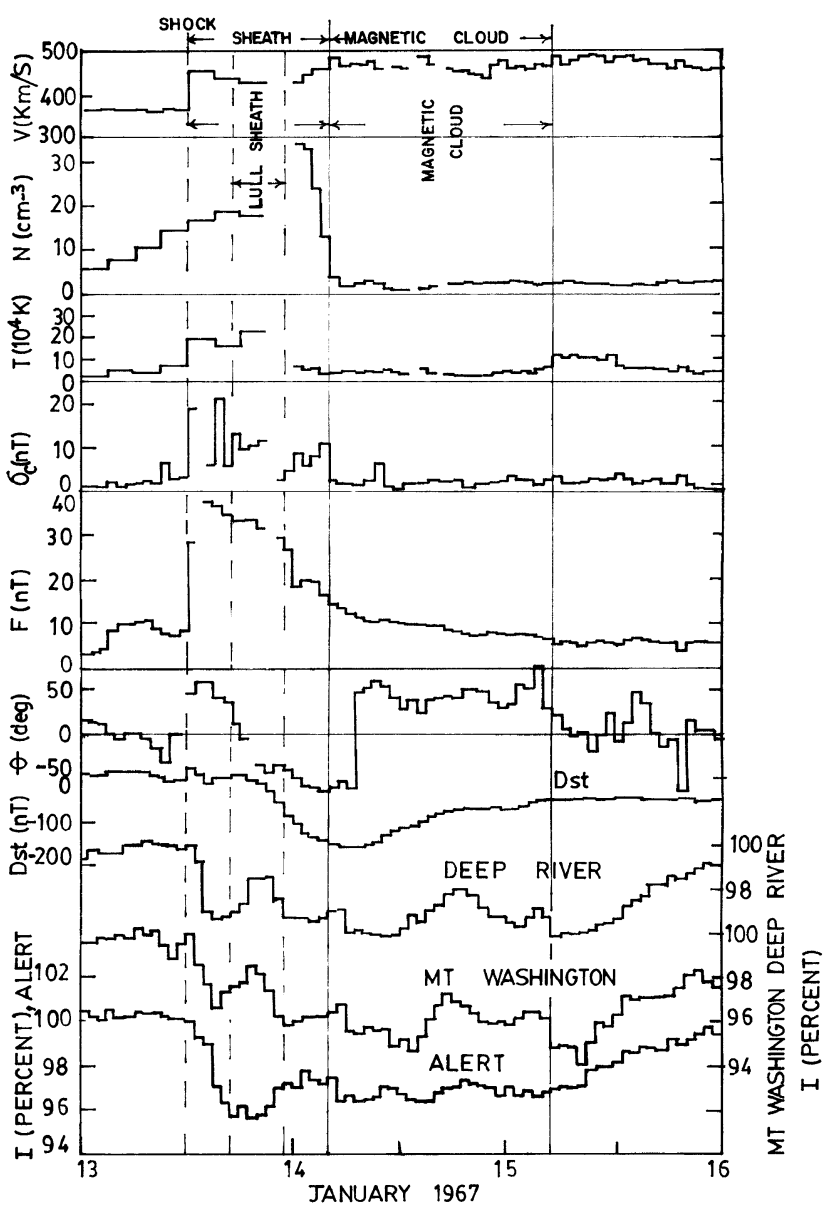

Fig. 3. Hourly profiles of the Deep River, Mount Washington and Alert nucleonic intensity (I), bulk-speed (V), density (N) and temperature (T) of the solar wind plasma, interplanetary magnetic field (IMF) magnitude (F) and latitude $(\theta)$ as well as the rms of the IMF components $\left(\sigma_{c}\right)$ and the geomagnetic activity Dst index on 13-15 January 1967 . The vertical dashed line, labeled SHOCK, marks the arrival of the interplanetary shock wave at the Earth. Though the bulk speed, density, and temperature data from 0100 to 2100 UT on 13 January appear to be 3 hourly averages, they are indeed the hourly average data listed in King (1977) as well as in CDAWeb. The single-step main phase storm is due to the combination of the sheath and the leading field of the magnetic cloud.

tector before the decrease and $N_{F}$ is the hourly average rate during the maximum depression of the cosmic ray intensity. Clearly, not only is the enhanced diurnal variation minimal in the Mount Washington and Deep River neutron monitor data in Fig. 5, but a relatively high ( $\gtrsim 10 \mathrm{nT}) \mathrm{rms}$ of the IMF components prominently separates both the Lull region and the onset of the second decrease of the two-step FD, from the magnetic cloud, in Figs. 1-5. The latter is certainly a diagnostic feature in for instance determining the front boundary of the magnetic cloud, since the rms of the IMF components is relatively low, $\lesssim 1 \mathrm{nT}$ and $\lesssim 5 \mathrm{nT}$ respectively, in the magnetic cloud and Lull regions. This is a new and objective criterion for the front boundary of the magnetic cloud, if we note that Burlaga (1991) stated that "determining an objective criterion for the boundary of a magnetic cloud is one of the most important problems for future research in magnetic clouds." Notice also that the IMF and SWP data in the magnetic cloud region, in Figs. 1-5, show a high field strength $(\gtrsim 10 \mathrm{nT})$ and rotation as well as a relatively low 


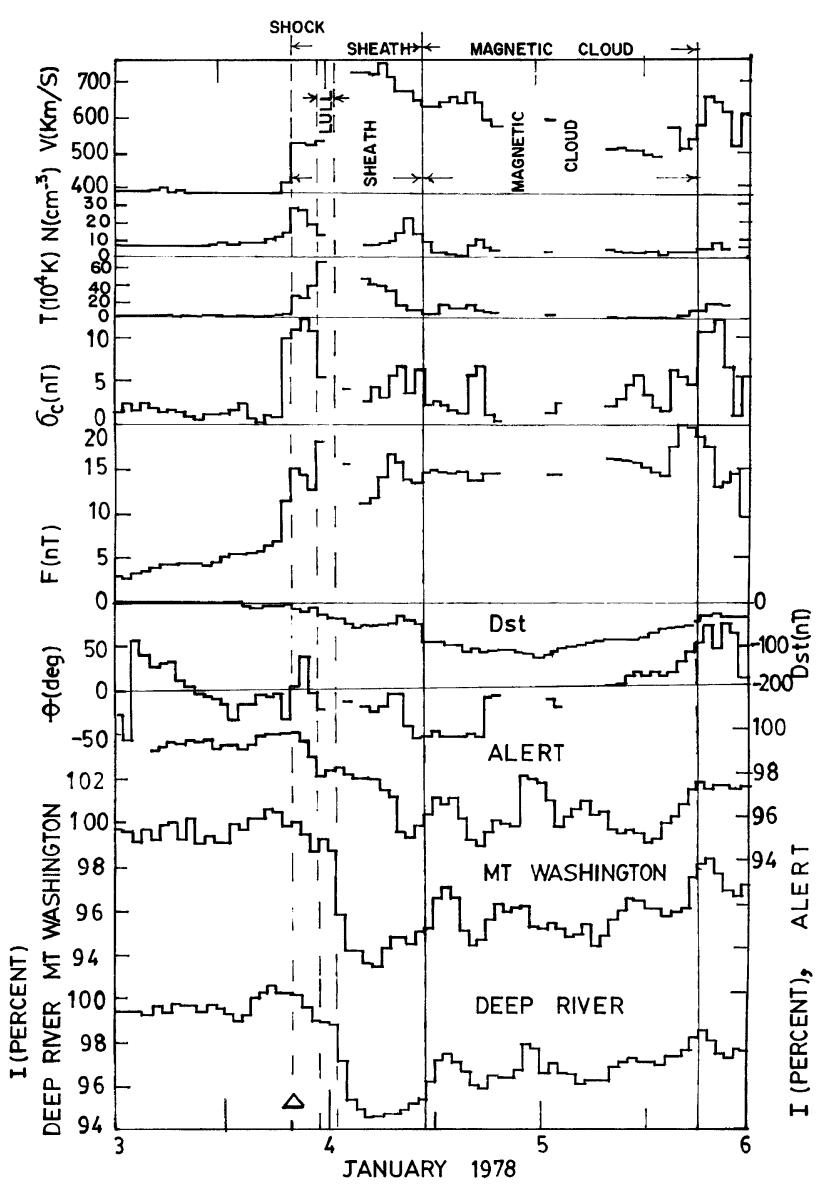

Fig. 4. Hourly profiles of the Alert, Mount Washington, and Deep River nucleonic intensity $(\mathrm{I})$, bulk-speed $(\mathrm{V})$, density $(\mathrm{N})$ and temperature $(\mathrm{T})$ of the solar wind plasma, interplanetary magnetic field (IMF) magnitude (F) and latitude $(\theta)$ as well as the rms of the IMF components $\left(\sigma_{c}\right)$ and geomagnetic activity Dst index on 3-5 January 1978. Storm sudden commencement is marked by a triangle, and the vertical dashed line, labeled SHOCK, marks the arrival of the interplanetary shock wave at the Earth. The two steps of the two-step main phase storm all begin in the sheath, while the second step continues in the leading region of the magnetic cloud.

density and temperature $\left(\lesssim 10^{4} \mathrm{~K}\right)$ and a relatively low $(\lesssim 1$ $\mathrm{nT}$ ) rms of the IMF components, which are the signatures of the magnetic cloud. The next five columns respectively give the start and end times as well as the maximum latitude of the IMF southern orientation, and the maximum and average values of the IMF total intensity during the southwardoriented IMF. And the last three columns respectively give the start and end times as well as the maximum values of the Dst Main Phase.

Also indicated in Table 1 are the locations (in terms of Magnetic Cloud, Lull, and Front and Rear Sheath regions) of the maximum SWP bulk speed as well as of the periods and largest values of both the IMF South Latitude and Dst Main Phase.

The sheath extends from shock time to the beginning of cloud region, in Table 1 and Figs. 1-5, and is sufficiently turbulent, as indicated by the large variations in both the field strength and direction as well as by the relatively high $(\gtrsim 10 \mathrm{nT}) \mathrm{rms}$ of the IMF components in the region, is hot $\left(\gtrsim 10^{5} \mathrm{~K}\right)$ and dense $\left(\gtrsim 10 \mathrm{~cm}^{-3}\right)$, and was most probably produced by the shock propagation in space. The large IMF

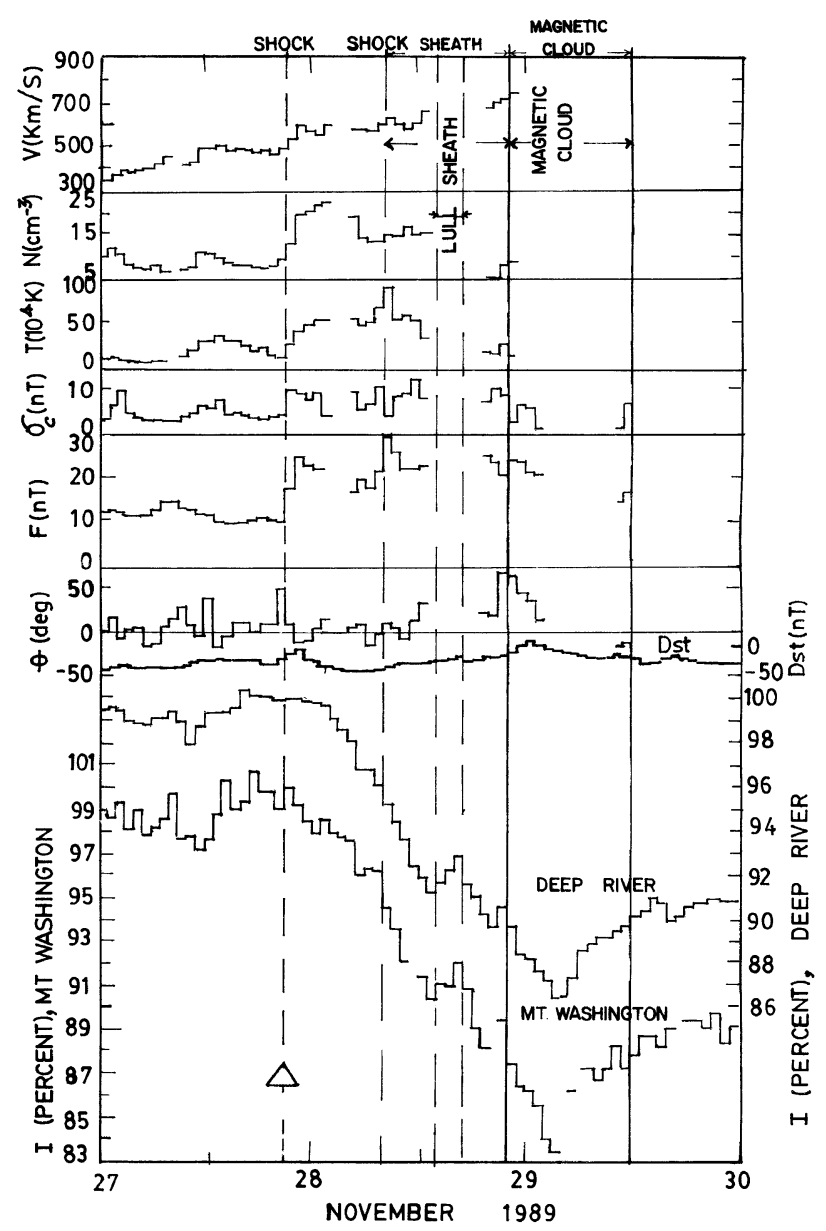

Fig. 5. Same as Fig. 4, but for Deep River and Mount Washington on 27-29 November 1989, and the Dst Main Phase during the period, of $\sim-30 \mathrm{nT}$, which is the background Dst value.

variations in the sheath scatter the galactic cosmic rays and thus sweep away the cosmic ray particles. When the IMF variations in the sheath become too feeble, the Lull region where the rms of the IMF components is relatively low $(\lesssim 5$ nT) as in Figs. 1-5, the scattering of the galactic cosmic rays ceases and the first step of the FD recovers. But the particle scattering resumes when the high IMF variations in the sheath resume and the onset of the second decrease starts. Also, the SWP bulk speed in both the sheath and magnetic cloud is much higher than the ambient value.

The first step of the two-step FD occurs in the Front Sheath while the second step begins in the Rear Sheath, but continues in the Magnetic Cloud where the galactic cosmic rays are also scattered away from the high magnetic field pressure in the magnetic cloud. The first step recovers in the Lull region, a region that is sandwiched between the Front and Rear Sheath as in Figs. 1-5. We emphasize that in the Front and Rear Sheath, the IMF variations are high as indicated by the relatively high ( $\gtrsim 10 \mathrm{nT}) \mathrm{rms}$ of the IMF components, but in the Lull and Magnetic Cloud regions, the IMF variations are relatively low as indicated by the relatively low $(\lesssim 5 \mathrm{nT}) \mathrm{rms}$ of the IMF components.

In Fig. 1, the single-step main phase storm starts at 0700 UT on September 29, 1978 in the Lull region of the Sheath and ends at 1500 UT on September 29 in the leading region 


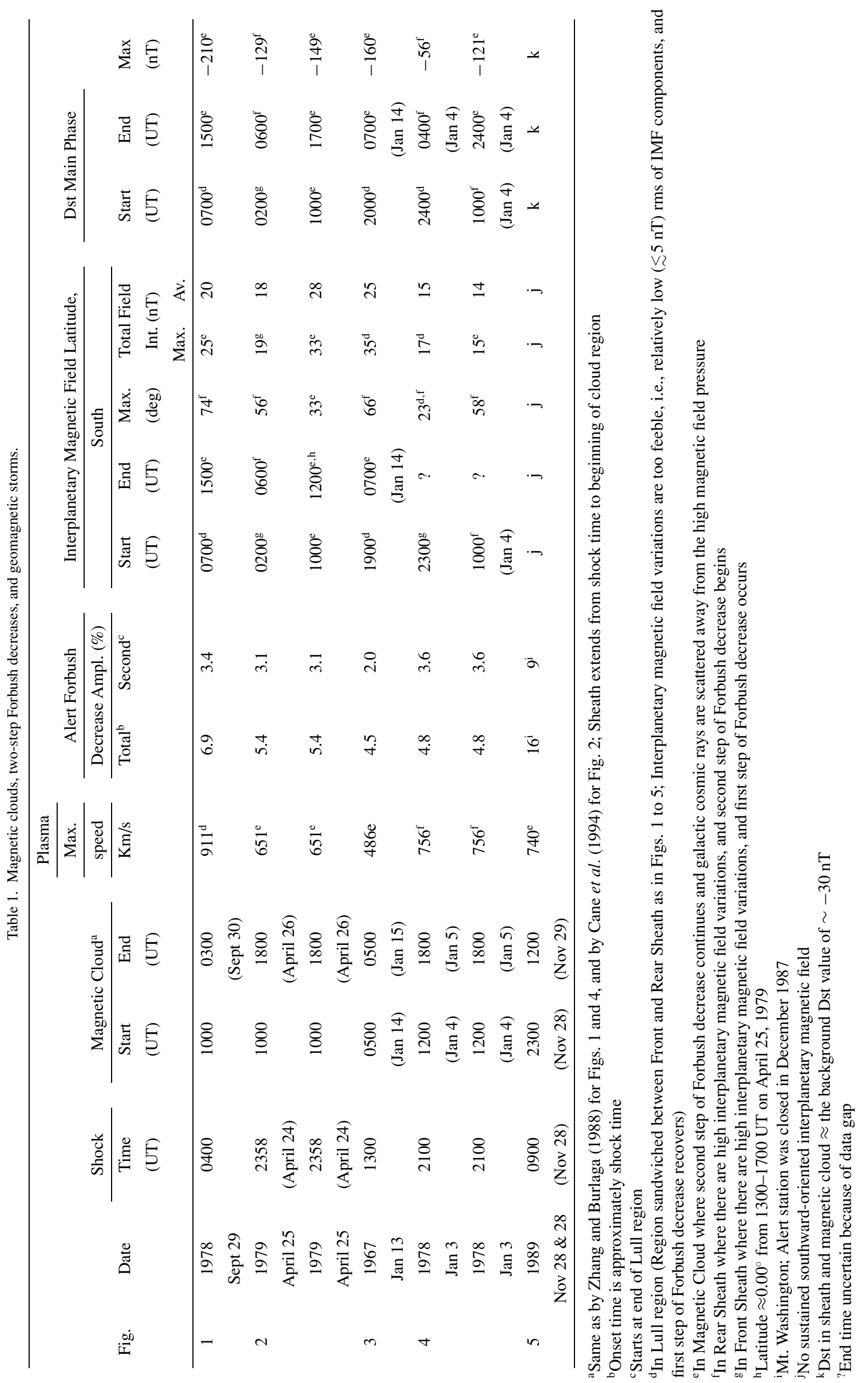


of the Magnetic Cloud, with maximum Dst Main Phase of $-224 \mathrm{nT}$, the start and the end times exactly coinciding with the period of IMF southern orientation. Notice that the Dst Main Phase is a smooth continuous curve with the deepest Dst Main Phase of $-210 \mathrm{nT}$ at 1500 UT on 29 September 1978, apart from the lone data point $-224 \mathrm{nT}$ at $1000 \mathrm{UT}$ on 29 September 1978, which is probably a flash in the pan.

In Fig. 2, the first step of the two-step main phase storm starts at 0200 UT on April 25, 1979 in the Front Sheath and ends at 0600 UT on April 25 in the Rear Sheath, with maximum Dst Main Phase of $-129 \mathrm{nT}$, the start and end times exactly coinciding with the first period of IMF southern orientation. The second step starts at 1000 UT on April 25, 1979 in the leading region of the Magnetic Cloud and ends at 1700 UT on April 25 also in the leading region of the Magnetic Cloud, with maximum Dst Main Phase of -149 $\mathrm{nT}$, the start and end times again coinciding with the second period of the IMF southern orientation, since the IMF latitude was $\approx 0.00^{\circ}$ from $1300-1700$ UT on April 25, 1979.

In Fig. 3, the single-step Dst Main Phase storm starts at 2000 UT on January 13, 1967 in the Lull region and ends at $0700 \mathrm{UT}$ on January 14 in the leading region of the Magnetic Cloud, with maximum Dst Main Phase of -160 $\mathrm{nT}$, the start and end times approximately coinciding with the period of the IMF southern orientation.

Finally, in Fig. 4, the first step of the two-step main phase storm starts at 2400 UT on January 3, 1978 in the Lull region, and ends at 0400 UT on January 4 in the Rear Sheath, with maximum Dst Main Phase of $-56 \mathrm{nT}$, the start time approximately coinciding with the start time of the first IMF southern orientation. The second step starts at 1000 UT on January 4 in the Rear Sheath and ends at 2400 UT on January 4 in the leading region of the Magnetic Cloud, with maximum Dst Main Phase of $-121 \mathrm{nT}$, the start time approximately coinciding with the start time of the second IMF southern orientation. The end times of the IMF southern orientation for both steps are uncertain because of the IMF data gap.

However, in Fig. 5 from 27 to 29 November 1989 during which there was no sustained IMF southern orientation, the Dst Main Phase was $\sim-30 \mathrm{nT}$, which was the background Dst Main Phase, even though the associated FD event, in Fig. 5, is a classic two-step FD with a record-breaking amplitude (e.g., 16\% at Mount Washington station).

\section{Discussion and Conclusions}

Table 1 and Figs. 1-4 show that Dst Main Phase starts at approximately the time that the IMF starts to turn South and ends approximately when the IMF starts to turn North. But in Fig. 5, where there is no sustained southward-oriented IMF, the Dst in the sheath and magnetic cloud, is approximately the background Dst for the period, of $\sim-30 \mathrm{nT}$, even though the associated FD event in Fig. 5 is a classic two-step FD with a record-breaking amplitude, as shown in Table 1. This is an indication that the southward-oriented IMF is the major source in triggering geomagnetic disturbances, by allowing a strong coupling between the solar wind and the magnetosphere, as first suggested by Dungey (1961).

Thus, the key role of the IMF North-South orientation in coupling the solar wind with the magnetosphere clearly emerges from Figs. 1-5. In addition, we observe that the initial set of the Dst Main Phase always begins in the sheath (which includes the Lull region) where, and when, the sustained southward-oriented IMF first occurs, but ceases when the IMF is rotated to a strong northward-orientation, only to resume at subsequent sustained southward-oriented IMF within the sheath and the leading (i.e. front) region of the magnetic cloud. This finding is important for space weather forecasting. Thus, since it was found that Dst Main Phase commenced in each region of the sheath, including the Lull region where the IMF variations are too feeble and first step of the two-step FD recovers, the storm was not influenced by the large IMF variations in the sheath, which did sustain the depressions in the cosmic ray intensity during the first and second steps of the FD. This is a new result, and is different from the findings of Zhang et al. (2004) that the multistep storms due to the leading field and the trailing field of a magnetic cloud are "probably driven by fluctuations in their southward field regions". However, it is well known that the IMF variations are weak in a magnetic cloud.

Wu and Lepping (2002), and Zhang et al. (2004) also investigated the regions of sheath-magnetic cloud structure that drive geomagnetic storms. Wu and Lepping (2002) found that a geomagnetic storm can be induced by a sheath, the leading region or the trailing region of a magnetic cloud, and both sheath and cloud regions during low solar activity years (1995-1998), and a multistep main phase storm could be caused by the combination of a sheath and cloud structure. On the other hand, Zhang et al. (2004) found that a storm can be driven by a cloud's various regions or their combinations with dissimilar occurrence percentages, the cloud's leading field was the most geoeffective region and the sheath region was equally effective at causing magnetic storms during the solar maximum years (January 1998-April 2002), and the multistep development storm can result not only from both the combination of sheath and cloud fields but also from different fields within a cloud. This is different from the results of Tsurutani and Gonzalez (1997), Gonzalez et al. (2001), and Wu and Lepping (2002), in which multistep main phase development storms can only be due to the combinations of sheath and cloud fields. However, as earlier indicated in the last section, we showed for the first time that multistep storms can also result from different component fields within the sheath itself (see Fig. 4), and that the sheath and the leading region of a magnetic cloud can also together produce a single-step storm (see Figs. 1 and 3). Also our studies here demonstrate that during maximum solar activity years (1967, 1978, 1979, and 1989) in solar cycles 20, 21, 22, the initial set of the Dst Main Phase always began in the sheath where, and when, the sustained southward- oriented IMF first occurred but ceased when the IMF was rotated to a strong northward-orientation, only to resume at subsequent sustained southward-oriented IMF within the sheath and the leading region of the magnetic cloud.

Thus, our studies have shown that the initial set of storms always begins in the sheath, which is an important result for space weather forecasting, and that what appears to 
determine the start and end times of a storm and/or each step of a storm is the key role of the North-South orientation in coupling the solar wind with the magnetosphere.

Also, it appears, from Figs. 1-4, and Table 1, that the larger the IMF south latitude in the sheath and magnetic cloud, the deeper the Dst index. For instance, the largest IMF south latitude of $74^{\circ}$ is in Fig. 1, and gives the deepest Dst index of $-210 \mathrm{nT}$, apart from the lone data point -224 nT, at 1000 UT on 29 September 1978, which though still deeper, but, does not fall on the smooth continuous Dst Main Phase curve, and the second largest south latitude of $66^{\circ}$ is in Fig. 3 and gives the second deepest Dst index of $-160 \mathrm{nT}$.

However, the third largest south latitude of $58^{\circ}$ is in Fig. 4 and gives the Dst index of $-121 \mathrm{nT}$, while the fourth largest south latitude of $56^{\circ}$ is in Fig. 2, but gives a slightly deeper Dst index of $-129 \mathrm{nT}$. It is possible that SWP speed is an important control on the Dst index, since during geomagnetic storms, the magnetopause is pushed toward the Earth, sometimes below the geostationary spacecraft orbit (6.6 Earth radii), and since the depth of minimum Dst appears to be influenced by the speed of the magnetic cloud (Zhang and Burlaga, 1988) and speed correlated with geomagnetic activity (e.g., Baker et al., 1984). Also, the higher solar wind dynamic pressure (enhanced magnetopause current) is understood to give positive Dst value. For the SWP bulk speed to be a contributory factor to Dst index, we would expect the speed in Fig. 4 to be larger than that in Fig. 2, since a larger speed would act to further compress the magnetosphere and thus give an additional positive value of Dst index, an expectation which is consistent with the observed maximum SWP bulk speed of $651 \mathrm{~km} / \mathrm{s}$ and 756 $\mathrm{km} / \mathrm{s}$ in Figs. 2 and 4 respectively, as shown in Table 1. On the other hand, in Fig. 2, where the SWP bulk speed has a rather constant value of $\sim 600 \mathrm{~km} / \mathrm{s}$ during the southwardoriented IMF, the south latitude of $56^{\circ}$ gives Dst index of $-129 \mathrm{nT}$, while the south latitude of $33^{\circ}$ gives Dst index of $-149 \mathrm{nT}$, an observation which cannot be explained using SWP speed as a contributory factor to Dst index.

Nevertheless, in Fig. 2, the IMF maximum and average intensities during the southward oriented IMF, as shown in Table 1 , are $19 \mathrm{nT}$ and $18 \mathrm{nT}$ for $56^{\circ}$ south latitude but attain the high values of $33 \mathrm{nT}$ and $28 \mathrm{nT}$ for $33^{\circ}$ south latitude, which is consistent with the respective observed values of Dst index of $-129 \mathrm{nT}$ and $-149 \mathrm{nT}$, since a higher IMF intensity could lead to a deeper Dst index. This finding is confirmed in Figs. 2 and 4 for the respective south latitudes of $56^{\circ}$ and $58^{\circ}$, where the IMF maximum and average intensities during the southward-oriented IMF, as shown in Table 1, are $19 \mathrm{nT}$ and $18 \mathrm{nT}$ in Fig. 2 and the lower values of $15 \mathrm{nT}$ and $14 \mathrm{nT}$ in Fig. 4, and are therefore consistent with the observed values of Dst index of $-129 \mathrm{nT}$ and $-121 \mathrm{nT}$ in Figs. 2 and 4, respectively. And finally, the least value of the IMF south latitude of $23^{\circ}$ is in Fig. 4 and gives the shallowest Dst index of -56 nT during the sustained IMF southern orientation. Clearly, the IMF intensity during the IMF southern orientation is a contributory factor to Dst index. Previous studies by Gonzalez and Tsurutani (1987) showed that intense storms with peak $D s t<-100 \mathrm{nT}$ are primarily caused by large
$B_{z}<-10 \mathrm{nT}$ fields with duration greater than 3 hours. In addition, our studies here clearly demonstrate that, even for intense storms, a higher IMF intensity during the sustained IMF southern orientation leads to a deeper Dst index, i.e., to a more intense storm.

Thus, we conclude that, due to ICME impacting on slow solar wind, there is a sheath upstream of the ICME led by a fast forward shock. And the large IMF variations in this sheath, as well as the high magnetic field pressure in the magnetic cloud, were observed to sustain the depressions in the cosmic ray intensity during FDs, while the southward IMF in the said sheath and magnetic cloud was found to be the major source in triggering geomagnetic storms, by allowing a strong coupling between the solar wind and the magnetosphere. It was also observed that the initial set of the Dst Main Phase always began in the sheath, which includes the Lull region, (a useful result for space weather forecasting) where, and when, the sustained southwardoriented IMF first occurred, but ceased when the IMF was rotated to a strong northward-orientation, only to resume at subsequent sustained southward-oriented IMF within the sheath and the leading region of the magnetic cloud. Since it was found that the main phase storm commenced in each region of the sheath, including the Lull region where the IMF variations are too feeble and the first step of the twostep FD recovers, the storm was not influenced by the large IMF variations in the sheath, which did sustain the depressions in the cosmic ray intensity during the first and second steps of the FD. This is another new result. We also, in the last section, showed for the first time, that the front boundary of the magnetic cloud is well defined by the relatively high ( $\gtrsim 10 \mathrm{nT}$ ) rms of the IMF components, which prominently separates both the Lull region and the onset of the second decrease of the two-step FD, from the magnetic cloud. There were some instances where a two-step main phase storm, caused by the combination of a sheath and cloud structure, occurred, the two steps sometimes both starting in the sheath itself, which is a new result. Also new is our finding that, in some instances, the sheath and the leading region of the magnetic cloud together produced a single-step storm. In addition, enhanced IMF south latitude and IMF intensity in the sheath and magnetic cloud during the IMF sustained southern orientation, were each observed to produce enhanced geomagnetic activity, even for intense storms. And high SWP bulk speed was found to reduce the depth of the Dst index.

Our results can be used to develop a reliable model on geomagnetic storms, since the presently available models (e.g., Burton et al., 1975; Pudovkin et al., 1988; Grafe, 1988; Feldstein, 1992; Gonzalez et al., 1989, 1994; Valdivia et al., 1996; O’Brien and McPheron, 2000) are controversial and discrepant. For instance, there is presently a great deal of controversy and discrepancy in determining the values of the relaxation time and the coupling function of Dst with the solar wind. Also, the ring current effect in the storm time depression is controversial with the estimates of the symmetric ring current contribution to Dst ranging from 0 to $40 \%$, and the contributions of the crosstail and partial ring current to Dst are very discrepant with the corresponding estimates varying from $15 / 25$ to $80 \%$ 
(Maltsev, 2003). Moreover, the Physics of the associated storm-substorm relationship is presently poorly understood (e.g., Diego et al., 2005, and references therein).

However, we infer from our results that, when the magnetosphere is exposed to a sustained southward-oriented IMF in the magnetic cloud and the sheath preceding it, a valve (i.e., valve-like IMF direction) opens and allows direct transfer of energy between the solar wind and the magnetosphere to trigger the geomagnetic storms. That is, the direction of the IMF in the magnetic cloud and the sheath acts like a valve that controls energy input from the solar wind into the magnetosphere, with the most favorable direction of the IMF, for triggering geomagnetic storms, being southward, which allows a strong coupling between the solar wind and the magnetosphere. For instance, the IMF is thus connected well, or merged, with the magnetic field of the Earth, allowing solar wind plasma and energy to directly enter, rather than flow around, the magnetosphere. The stronger the sustained IMF southward-orientation, the wider the valve opens, the higher the SWP bulk speed, the narrower the opening in the valve becomes. And the more the IMF strength during the IMF southern orientation, the larger is the solar wind energy density that is available for transfer through the valve. The valve closes when the IMF is rotated to a strong northward-orientation, and the geomagnetic storms cease.

Acknowledgments. The author would like to thank Prof. J. A. Lockwood, Drs. G. Dolling (Atomic Energy of Canada Limited) and E. Erwin (National Geophysical Data Center, U.S.A.) for their assistance with the neutron monitor data, Dr. J. H. King and the National Space Science Data Center, U.S.A. for providing the interplanetary magnetic field and solar wind plasma data, and the referees for their very valuable comments.

\section{References}

Baker, D. N., S. I. Akasofu, W. Baumjohann, J. W. Bieber, D. H. Fairfield, E. W. Hones, Jr., B. Mauk, R. L. McPherron, and T. E. Moore, Substorms in the magnetosphere, in Solar Terrestrial Physics, Present and Future, NASA Ref Pubs. 1120, edited by D. M. Butler and K. Papadupoulos, p. 8, NASA, Washington, D.C., 1984.

Bavassano, B., M. Storini, N. Iucci, and M. Parisi, Some properties of socalled magnetic cloud structures in the solar wind, Adv. Space Res., 9, 63-68, 1989.

Burlaga, L. F., Magnetic Clouds, in Physics of the Inner Heliosphere, Vol. 2, edited by R. Schwenn and E. Marsch, Springer-Verlag, New York, 1991.

Burlaga, L. F., Sittler, F. Mariani, and R. Schwenn, Magnetic loop behind an interplanetary shock: Voyager, Helios and IMP 8 observations, $J$. Geophys. Res., 86, 6673-6684, 1981.

Burton, R. K., R. L. McPherron, and C. T. Russel, An empirical relationship between interplanetary conditions and Dst, J. Geophys. Res., 80, 4204-4214, 1975.

Cane, H. V., I. G. Richardson, T. T. von Rosenvinge, and G. Wibberenz, Cosmic ray decreases and shock structure: A multispacecraft study, $J$. Geophys. Res., 49, 21429-21441, 1994.

Diego, P., M. Storini, M. Parisi, and E. G. Cordaro, AE index variability during corotating fast solar wind streams, J. Geophys. Res., 110, A06105, doi:10.1029/2004JA 010715, 2005.

Dungey, J. W., Interplanetary magnetic field and the auroral zones, Phys.
Rev. Lett., 6, 47-48, 1961.

Feldstein, Y. I., Modelling of the magnetic field of magnetospheric ring current as a function of interplanetary parameters, Space Sc. Revs., 59, 83-165, 1992.

Gonzalez, W. D. and B. T. Tsurutani, Criteria of interplanetary parameters causing intense magnetic storms (Dst $<-100 \mathrm{nT})$, Planet. Space Sci., 35, 1101-1109, 1987.

Gonzalez, W. D., B. T. Tsurutani, A. L. C. Gonzalez, E. J. Smith, F. Tang, and S. I. Akasofu, Solar wind-magnetosphere coupling during intense magnetic storms (1978-1979), J. Geophys. Res., 94, 8835-8851, 1989.

Gonzalez, W. D., J. A. Joselyn, Y. Kamide, H. W. Krochl, G. Rostoker, B. T. Tsurutani, and V. M. Vasyliunas, What is a geomagnetic storm?, $J$. Geophys. Res., 99, 5771-5792, 1994.

Gonzalez, W. D., A. L. C. Gonzalez, J. H. A. Sobral, A. D. Lago, and L. Vieira, Solar and interplanetary causes of very intense geomagnetic storms, J. Atmos. Sol. Terr. Phys., 63, 403-412, 2001.

Gosling, J. T., Coronal mass ejections: The link between solar and geomagnetic activity, Phys. Fluids, B, 5(7) 2638-2645, 1993a.

Gosling, J. T., The solar flare myth, J. Geophys. Res., 98, 18937-18949, 1993 b.

Grafe, A., The influence of the recovery phase injection on the decay of the ring current, Planet. Space Sci., 36, 765-773, 1988.

Ifedili, S. O., Forbush decrease of June 8, 1969: Causes of the unusually long recovery, Earth Planets Space, 53, 993-999, 2001.

Ifedili, S. O., The two-step Forbush decrease: An empirical model, J. Geophys. Res., 109, A002117, doi: 10.1029/2002JA009814, 1-12, 2004.

Iucci, N., M. Parisi, C. Signorini, M. Storini, and G. Villoresi, Short-term cosmic ray increases and magnetic cloud-like structures during Forbush decreases, Astron. Astrophys. Suppl. Ser., 81, 367-391, 1989.

King, J. H., Interplanetary Medium Data Book, Rep. NSSDC/WDC - A - R \& S 77 - 04a, NASA Goddard Space Flight Cent., Greenbelt, Md, 1977.

King, J. H., Interplanetary Medium Data Book, Rep. NSSDC/WDC - A R \& S 83 - 01, NASA Goddard Space Flight Cent., Greenbelt Md, 1983.

King, J. H. and N. E. Papitashvili, Interplanetary Medium Data Book, Rep. NSSDC/WDC - A - R \& S 94 - 08, NASA Goddard Space Flight Cent., Greenbelt, Md, 1994

Klein, L. W. and L. F. Burlaga, Interplanetary magnetic clouds at $1 \mathrm{AU}, J$. Geophys. Res., 89, 613-624, 1982.

Maltsev, Y. P., The points of controversy in magnetic storm study (Review), Proc. XXVI Annual Seminar "Physics of Auroral Phenomena", Apatity, Russia, pp. 33-40, 2003.

O'Brien, T. P. and R. L. McPheron, An empirical phase space analysis of ring current dynamics: Solar wind control of injection and decay, $J$. Geophys. Res., 105, 7707-7719, 2000.

Pudovkin, M. I., A. Grafe, S. A. Zaitseva, L. Z. Sizova, and A. V. Usmanov, Calculating the Dst-variation field on the basis of solar wind parameters, Gerlands Beitr. Geophysik., 97(6), 525-533, 1988.

Storini, M., Galactic cosmic-ray modulation and solar-terrestrial relationships, Nuovo Cimento Soc. Ital. Fis, C, 13, 103-124, 1990 (Errata corrige, Nuovo Cimento Soc. Ital. Fis. C, 14, 211, 1991.)

Tsurutani, B. T. and W. D. Gonzalez, The interplanetary causes of magnetic storms, in Magnetic Storms, Geophys. Monogr. Ser. Vol. 98, edited by B. T. Tsurutani et al., pp. 77-89, AGU, Washington, D.C., 1997.

Valdivia, J. A., A. S. Sharma, and K. Papadopoulos, Prediction of magnetic storms by nonlinear models, Geophys. Res. Lett., 23, 2899-2902, 1996.

Wu, C. C. and R. P. Lepping, Effects of magnetic clouds on the occurrence of geomagnetic storms: The first 4 years of Wind, J. Geophys. Res., 107(A10), 1314, doi: 10.1029/2001JA000161, 2002.

Zhang, G. and L. F. Burlaga, Magnetic clouds, geomagnetic disturbances, and cosmic ray decreases, J. Geophys. Res., 93, 2511-2518, 1988.

Zhang, J., M. W. Liemohn, J. U. Kozyra, B. J. Lynch, and T. H. Zurbuchen, A statistical study of the geoeffectiveness of magnetic clouds during high solar activity years, J. Geophys. Res., 109, A09101, doi: 10.1029/2004JA010410, 1-12, 2004.

S. O. Ifedili (e-mail: sifedili@yahoo.com) 